\title{
CO-ORDINATED INTERDISCIPLINARY EFFORTS ON RESEARCH IN ANIMAL PRODUCTION AND HEALTH
}

\author{
Hans Houe \\ Research Centre for the Management of Animal Production and Health \\ Foulum, 8830 Tjele
}

Co-ordinated interdisciplinary efforts on research in animal production and health. The objectives are to review results and experiences from interdisciplinary research projects in Research Centre for the Management of Animal Production and Health (CEPROS) concerning scientific content, organisation and collaboration.

The Centre has been founded as a result of an agreement between four institutions: the Danish Institute of Agricultural Sciences (DIAS), the Danish Veterinary Laboratory (DVL), the Danish Veterinary Institute for Virus Research (DVIV) and the Royal Veterinary and Agricultural University (KVL). CEPROS is a "research centre without walls" and is physically located as an integrated part of the four institutions named above. The Centre has close collaboration with the industry.

The superior goals of the Centre are to co-ordinate fundamental and applied research and simultaneously integrate the veterinary and the production oriented livestock research within animal health and welfare, taking into consideration the production economics and reduced use of medication. The assignment of the Centre is to initiate and carry out research, aiming to investigate the influence of breeding and production systems on animal health and welfare as well as on production and product quality.

The Centre has since 1997 established 16 interdisciplinary research projects dealing with cattle, pigs, poultry or mink. The scientific content can be divided into three research clusters: A. Management of animal production and health in production systems, B: Pathogenesis of production diseases and C. Animal health economics. In Cluster A, the physical environments of production systems have been investigated, broader definitions of the concept health have been established and used in identification of risk factors. Cluster B has investigated physiological, immunological and genetic mechanisms behind development of production diseases and how to apply this knowledge in disease prevention. The cluster in animal health economics has developed decision support tools for disease control in swine and cattle herds.

The interdisciplinary research groups organised in a centre without walls are found very useful for dealing with complex issues. The initial phases in the projects may be long as it takes time for different researchers to define common goals and identify methods. The individual researcher must get some insight in other disciplines without loosing his/her own area of expertise. In order to reach a synthesis of the different parts of a project, it is important that the project is well organised and there is participation of researchers with both specialised insight and researchers with a more broad insight in the problems. It is also important that the integration process is balanced and not too many research disciplines are included in the same project. 